О.А. ПУстотИНА, д.М.Н., профессор, Российский университет дружбы народов, Москва

\title{
ПРЕГРАВИДАРНАЯ ПОДГОТОВКА
}

В статье представлены основные положения прегравидарной подготовки. Обоснована целесообразность диагностических и лечебно-профилактических мероприятий при подготовке к беременности, а также роль планирования семьи в профилактике акушерских и перинатальных осложнений.

Ключевые слова: прегравидарная подготовка, планирование семьи, профилактика перинатальных осложнений, ДНТ, фолиевая кислота.

\section{O.A. PUSTOTINA, MD, Prof., Russian University of People's Friendship, Moscow PRECONCEPTION PREPARATION}

The article provides major provisions of the preconception preparation. The practicability of diagnostic and therapeutic and preventive measures at preparation to pregnancy as well as the role of the family planning in prevention of obstetrical and perinatal complications is justified.

Keywords: preconception preparation, family planning, prevention of perinatal complications, DWNT, folic acid

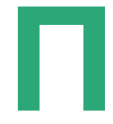
регравидарная подготовка (лат. gravida беременная, pre - предшествие), отечественный аналог преконцепционной подготовки (англ. conception - зачатие), включает комплекс диагностических и лечебно-профилактических мероприятий, направленных на подготовку супружеской пары к полноценному зачатию, вынашиванию беременности и рождению здорового ребенка. Преконцепционная оценка факторов риска и коррекция выявленных нарушений - наилучший доказанный способ снижения акушерских и перинатальных осложнений [1, 2]: материнской и перинатальной смертности, материнской и неонатальной заболеваемости, распространенности врожденных пороков развития (ВПР) и хромосомных аномалий у плода, частоты самопроизвольных выкидышей и преждевременных родов, а также социальных и экономических последствий для общества, связанных с выхаживанием и реабилитацией недоношенных детей и детей-инвалидов. В 2016г. был опубликован клинический протокол «Прегравидарная подготовка» [3], отражающий консенсус экспертов по вопросам подготовки супружеской пары к беременности в различных клинических ситуациях, основанный на изучении отечественной и мировой доказательной базы. Документ прошел два общественных слушания в рамках общероссийских конференций; над его созданием и совершенствованием работали более 60 специалистов из разных областей медицины.

\section{Общие положения прегравидарной подготовки}

- Рационально спланированная заблаговременная прегравидарная подготовка значительно снижает вероятность рождения детей с ВПР: дефектами нервной трубки (ДНТ), пороками сердца, челюстно-лицевыми пороками и хромосомными аномалиями.

- Коррекция дефицита микронутриентов у будущей матери значительно улучшает соматическое здоровье, когнитивные способности и продолжительность жизни ее ребенка.

चрегравидарное консультирование необходимо проводить всем женщинам репродуктивного возраста на любом плановом и внеплановом визите к акушеру-гинекологу. Врачи любых специальностей должны также учитывать возможность наступления беременности у таких женщин и информировать о предполагаемых рисках для их здоровья, плода и новорожденного как самого заболевания, так и проводимого лечения.

- Прегравидарное обследование и коррекция выявленных заболеваний женщины снижают риск осложнений беременности, родов и послеродового периода.

- Репродуктивное просвещение и прегравидарное консультирование - безальтернативные методы повышения информированности населения по вопросам сохранения репродуктивного здоровья, профилактики абортов и приверженности планирования и подготовки беременности.

- В интересах сохранения здоровья матери и ребенка интергенетический интервал должен составлять не менее $24+9$ мес. (время от родоразрешения до зачатия плюс 9 мес. беременности). При наличии экстрагенитальных заболеваний оптимальная длительность интергенетического интервала зависит от сроков их компенсации. Единственный путь гарантированного обеспечения оптимального периода между беременностями - рациональная контрацепция.

- Наилучший период для наступления последующей беременности после самопроизвольного аборта до 12 нед. - 3-6 мес.

- Акушерский и перинатальный риски минимальны у пациенток в возрастной группе 19-35 лет; беременность и роды у женщин моложе 19 и старше 35 лет сопряжены с повышением вероятности различных осложнений для матери и плода. 
Прегравидарная подготовка необходима обоим будущим родителям, поскольку они в равной мере обеспечивают эмбрион генетическим материалом и несут сопоставимую ответственность за здоровое зачатие и рождение здорового ребенка. При этом в минимальном объеме она необходима всем супружеским парам, планирующим беременность. Избежать избыточности позволяет дифференцированный подход, основанный на выделении групп высокого риска.

Прегравидарная подготовка необходима обоим будущим родителям, поскольку они в равной мере обеспечивают эмбрион генетическим материалом и несут сопоставимую ответственность за здоровое зачатие и рождение здорового ребенка

\section{Этапы прегравидарной подготовки:}

І. Определение состояния здоровья будущих родителей и оценка факторов риска:

с сбор анамнеза,

физикальное обследование,

лабораторный скрининг.

II. Профилактические мероприятия:

планирование беременности,

коррекция образа жизни (питания, веса, физической активности), отказ от вредных привычек,

дотация витаминов и микроэлементов.

III. Дополнительныелечебно-профилактические мероприятия у женщин с отягощенным акушерско-гинекологическим анамнезом и экстрагенитальными заболеваниями.

\section{І.БАЗОВЫЙ ОБЪЕМ ПЕРВИЧНОГО ОБСЛЕДОВАНИЯ}

\section{1. Сбор анамнеза}

Сбор общего анамнеза:

- перенесенные ранее заболевания, травмы или операции,

наличие хронических заболеваний,

- наличие наследственных заболеваний,

воздействие экологических факторов (радиация, химические агенты),

- условия труда и риски, связанные с профессиональной деятельностью,

бытовые условия, образ жизни,

- особенности пищевого поведения,

- приверженность вредным привычкам,

- прием медикаментов, противопоказанных беременным, аллергические реакции,

- семейный анамнез: заболевания с наследственной предрасположенностью (инсульт, инфаркт, артериальная гипертензия (АГ), онкологические заболевания, эндокринопатии).

Сбор репродуктивного анамнеза женщины:

- Менструальная функция:возрастменархе,длительность менструального цикла, регулярность и болезненность менструаций, обильность кровопотери.
Возраст начала половой жизни, сексуальная активность, количество половых партнеров и их смена за последние 6 мес., применяемые методы контрацепции (вид, длительность).

- Акушерский анамнез: число и исход предыдущих беременностей, рождение детей с ВПР, антенатальная гибель плода, эктопическая беременность, использование вспомогательных репродуктивных технологий (ВРТ), осложнения предыдущих беременностей - преэклампсия, АГ, гестационный диабет, преждевременные роды, задержка роста плода (ЗРП), отслойка плаценты.

- Наличие бесплодия: отсутствие беременности в течение 1 года при регулярной половой жизни без использования методов предохранения от беременности до возраста женщины 35 лет или 6 мес. - старше 35 лет; невынашивания беременности.

- Оперативные вмешательства на органах брюшной полости и малого таза, указания на осложнения после абортов и родов.

- Воспалительные заболевания органов малого таза (ВЗОМТ), инфекции, передаваемые половым путем (ИППП).

\section{2. Осмотр и физикальное обследование женшины}

- Измерение роста, индекса массы тела (ИМТ), артериального давления (АД), частоты дыхательных движений, частоты сердечных сокращений.

Общее физикальное обследование органов дыхания, кровообращения, пищеварения, мочевыводящей системы, опорно-двигательного аппарата, молочных желез.

- Гинекологический осмотр: влагалищное исследование при помощи зеркал со взятием мазков, бимануальное влагалищное исследование.

\section{3. Лабораторное и инструментальное обследование}

- Клинический анализ крови.

- Определение группы крови и резус-фактора (при отрицательном резус-факторе женщины необходимо уточнить резус-принадлежность партнера).

Определение сывороточных антител (АТ) к бледной трепонеме, вирусу иммунодефицита человека (ВИЧ), выявление HBsAg, HCVAg. Рутинный скрининг на вирус цитомегалии, простого герпеса и токсоплазму не проводится.

- Определение уровня глюкозы в плазме венозной крови (верхняя граница нормы 5,1 ммоль/л).

- Определение специфических IgG к вирусам кори и ветряной оспы (желательно).

- Общий анализ мочи.

- Исследование отделяемого женских половых органов на ИППП методом ПЦР (качественное исследование) для определения Chlamydia trachomatis, Neisseria gonorrhoeae, Trichomonas vaginalis, Mycoplasma genitalium.

- Цитологическое исследование мазков с шейки матки (РАР-тест).

- Трансвагинальное ультразвуковое исследование (УЗИ) органов малого таза.

- УзИ молочных желез/маммография (5-11-й день цикла). 
Дополнительный спектр лабораторного и инструментального скрининга, а также потребность в дополнительных консультациях смежных специалистов определяется индивидуально, ориентируясь на результаты базового обследования.

Прегравидарное обследование и коррекция выявленных заболеваний женщины снижают риск осложнений беременности, родов и послеродового периода

\section{ІІ.БАЗОВЫЙ ОБЪЕМ ПРОФИЛАКТИЧЕСКИХ МЕРОПРИЯТИЙ}

\section{Планирование беременности}

Необходимым условием благоприятного течения и исхода беременности является ее планирование. Данный подход дает возможность будущим родителям подготовиться к беременности, улучшить собственное здоровье, исключить или уменьшить воздействие факторов риска и произвести зачатие в наиболее оптимальный для них период.

Планирование семьи - это комплекс мероприятий, направленных на снижение количества нежеланных беременностей и числа абортов, на достижение оптимального интервала между беременностями и предупреждение деторождения у женщин моложе 19 и старше 35 лет с целью снижения риска материнской и младенческой заболеваемости и смертности [4].

Интервал между беременностями (англ. interpregnancy interval) - это временной интервал между датой зачатия и датой окончания предыдущей беременности. При планировании семьи также учитывают интергенетический интервал (лат. inter - между и греч. genesis - рождение)- период между последовательными родами. Оптимальным интергенетическим интервалом считается $24+9$ мес. (время от родоразрешения до зачатия плюс длительность беременности). Слишком короткий промежуток между последовательными родами, как и чрезмерное удлинение интергенетического интервала, значительно повышает риск ВПР, перинатальной смертности, преждевременных родов, рождения маловесных детей, материнской и неонатальной заболеваемости $[5,6]$.

Единственный путь гарантированного соблюдения оптимального интервала между родами - рациональная контрацепиия [7].

Наилучший период для наступления последующей беременности после самопроизвольного аборта до 12 нед. - 3-6 мес. По сравнению с более поздним наступлением беременности снижается частота повторного выкидыша, эктопической беременности, преждевременных родов, низкого веса при рождении, кесарева сечения и индуцированных родов [8].

К сожалению, в России, как и во всем мире, значительное количество (40\%) беременностей - незапланированные [9], при этом доля пар, прошедших прегравидарную подготовку, не превышает 4\% [10]. Это означает, что возможности большинства стратегий по предупреждению неблагоприятных исходов беременности (включая профилактику ВПР плода, преждевременных родов и преэклампсии) не используются или уже неэффективны. В целом прегравидарное консультирование следует рассматривать как реализацию конституционного права каждого гражданина РФ на охрану здоровья и медицинскую помощь, а также меру государственной поддержки семьи, материнства и детства.

Возраст женщины достоверно влияет на степень акушерского риска. Беременность в возрасте 15-19 лет сопряжена с повышенным риском анемии, преждевременных родов и преэклампсии в сравнении с беременными в возрасте 20-35 лет. Беременные старше 35 лет еще более подвержены акушерскому риску, в том числе АГ, гестационного диабета, отслойки плаценты. Риск материнской смертности в 35-39 лет в 2,5 раза выше, а после 40 лет - в 5,3 раза выше, чем у женщин 20-30 лет. После 35 лет увеличивается риск ВПР и хромосомных аномалий плода [11].

В целом около 2-3\% всех детей рождаются с различными аномалиями развития. Консультацию генетика при планировании беременности необходимо настоятельно рекомендовать супругам, имеющим в настоящем или предыдущих браках ребенка с ВПР или наследственными заболеваниями, а также при выявлении аномального кариотипа в остатках плодного яйца после досрочного прерывания беременности [2].

\section{Акушерский и перинатальный риски минимальны у пациенток в возрастной группе 19-35 лет; беременность и роды у женцин моложе 19 и старие 35 лет сопряжены с повышением вероятности различных осложнений для матери и плода}

АД. Регулярное измерение АД позволяет выявить женщин с АГ и включить их в группу риска осложнений беременности: преэклампсии, преждевременной отслойки плаценты, ЗРП, преждевременных родов. Стабилизация АД в преконцепционный период с помощью антигипертензивных средств значимо снижает риск акушерских и перинатальных осложнений [12].

Модификация образа жизни (питания, веса, физической активности)

Соблюдение режима дня, умеренные физические нагрузки, исключение стрессов и сбалансированный режим питания являются наиболее важными аспектами эффективности прегравидарной подготовки [2]. Оптимальный микронутриентный статус женщины перед зачатием достоверно положительно влияет на соматическое здоровье, когнитивные способности и продолжительность жизни будущего ребенка [13-15].

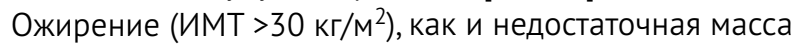

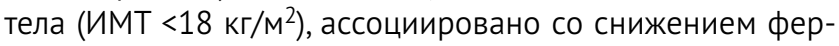
тильности и осложненным течением беременности. У женщин с ожирением рекомендована редукция массы тела путем коррекции питания, увеличения физической 
нагрузки и медикаментозной терапии. В противном случае значительно повышается риск таких осложнений беременности, как АГ, преэклампсия, гестационный диабет, аномалии родовой деятельности, кесарево сечение, послеоперационные осложнения, а также хромосомные аномалии у плода. Недостаточное питание вызывает дефицит витаминов, электролитов, приводит к сердечнососудистым и желудочно-кишечным заболеваниям, а также ассоциировано с высоким перинатальным риском (недоношенность, низкий вес при рождении, микроцефалия), поэтому перед планированием беременности женщинам с низким ИМТ необходимо повышение веса до достижения ИМТ $\geqslant 19$ кг/M² и более [13-15].

Следует обсудить с обоими будущими родителями негативное влияние табакокурения (в том числе пассивного вдыхания табачного дыма), злоупотребления алкоголем, употребления наркотических средств на сперматогенез, оогенез, возможность зачатия, вынашивание и исход беременности.

\section{Прегравидарная вакцинация}

Если пациентка до наступления беременности серонегативна в отношении вируса кори, краснухи и ветряной оспы (отсутствуют специфические IgG), то не менее чем за 3 мес. до зачатия необходимо провести вакцинацию [16]. Противопоказания к вакцинации: иммунодефицит, онкологические заболевания, аллергия на аминогликозиды и белок яйца, срок менее 3 мес. после внутривенного введения иммуноглобулина.

\section{Профилактика резус-конфликта}

Резус-конфликт возникает при беременности резусотрицательной матери резус-положительным плодом, зачатым от резус-положительного мужчины. Согласно Приказу М3 №572, женщину следует информировать о необходимости ежемесячного контроля резус-АТ в крови и на сроке 28 нед. ввести специфический антиD-иммуноглобулин с возможным повторным введением на сроке 34 нед. и обязательным - не позднее 72 ч после родов (при выявлении резус-положительного фактора у новорожденного).

\section{Дотация витаминов и микроэлементов}

Дотация фолатов. Всем женщинам, планирующим беременность, обязателен прием фолиевой кислоты в суточной дозе 400-800 мкг не менее чем за 1 мес. до наступления беременности и на протяжении I триместра. Дефицит фолатов вызывает нарушение эмбриогенеза и формирование ВПР у плода, в том числе ДНТ [17]. В европейских странах частота ДНТ составляет 100 на 100000 новорожденных, в странах Латинской Америки достигает 500, в России - 200-300 [18].

Также следствием дефицита фолатов являются аномалии конечностей, ушей, мочевыделительной системы, расщелина верхнего неба, омфалоцеле, часто диагностируют пороки сердечно-сосудистой системы [19].

В целом в Российской Федерации с 2000 по 2014 г. произошел беспрецедентный (на 75\%) рост частоты ВПР и хромосомных аномалий у плода: в 2000 г. их было зарегистрировано 659,5 на 100000 новорожденных, в 2014 г. - 1154,8 [20].
Дотация фолатов в периконцепционный период сокращает частоту ДНТ плода на 70\% [21], пороков сердца - на 26-40\% [22]. Дополнительный прием фолатов до и после зачатия достоверно снижает риск преждевременных родов, рождения маловесных детей, преэклампсии и отслойки плаценты [23-25], при этом только стартовая терапия фолатами в прегравидарном периоде позитивно влияет на течение и исход беременности, тогда как начало приема фолатов после наступления беременности такого эффекта не имеет [26].

С низким потреблением фолиевой кислоты в периконцепционный период ассоциирован низкий индекс психического развития у детей, когнитивные нарушения, повышенный риск аутизма и шизофрении [27]. Дотация фолатов во время беременности улучшает их двигательные, когнитивные и поведенческие показатели $[28,29]$.

Адекватное насыщение организма фолатами при ежедневном применении 400 мкг происходит в течение 8-12 недель [30]. В более короткие сроки восполнить дефицит возможно с увеличением их дозы. Так, прием 800 мкг фолиевой кислоты в сутки позволяет уже за 4 недели до зачатия достичь минимально достаточного (906 нмоль/л) ее уровня в эритроцитах для полноценного развития органов и систем будущего ребенка (рис. 1) [31].

\section{Рисунок 1. Достижение оптимального уровня фолатов В эритроцитах}

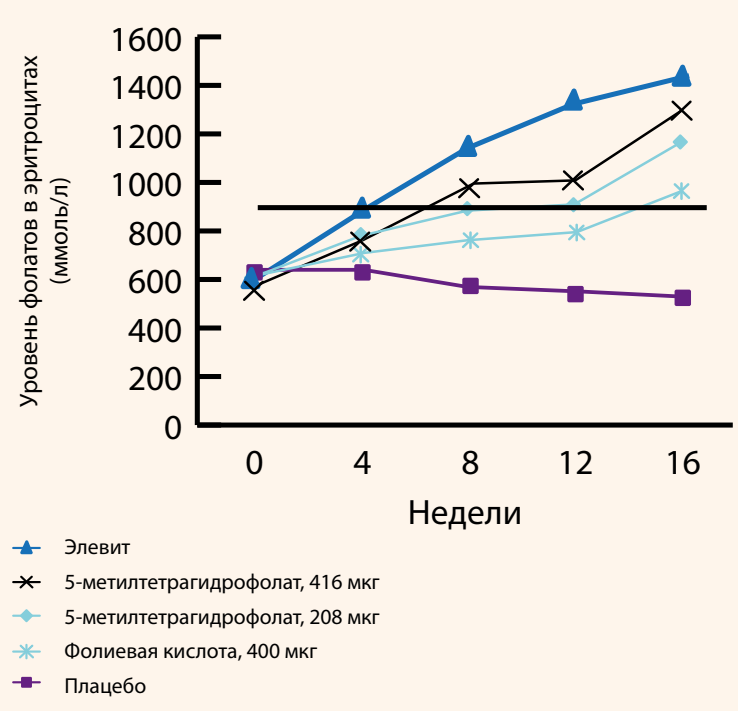

Назначение фолатов в составе витаминно-минеральных комплексов (ВМК) также эффективно снижает риск пороков развития у плода. Рандомизированное плацебоконтролируемое двойное слепое исследование показало, что прием ВМК (Элевит Пронаталь, содержащий 800 мкг фолиевой кислоты) у 2471 женщины в периконцепционный период на 93\% снижает частоту ДНТ у плода в сравнении с приемом плацебо у 2391 женщины [32]. Более того, достоверно снижается риск не только ДНТ, но и пороков сердечно-сосудистой системы, конечностей, мочеполовых органов и челюстно-лицевых аномалий 
$[33,34]$. Сочетанное применение фолиевой кислоты с другими витаминами группы B (B6, B12) улучшает метаболизм фолатов в организме, обеспечивая деятельность ферментов фолатного цикла, что особенно важно у женщин, которые имеют нарушения их функции вследствие генетического полиморфизма.

Более высокие дозы (4000-5000 мкг) фолатов в периконцепционный период назначают женщинам, имеющим высокий риск ДНТ у плода: ДНТ в анамнезе, сахарный диабет, прием антиконвульсантов, синдром мальабсорбции. При этом 400-800 мкг фолатов должно поступать в организм из ВМК (например, Элевит Пронаталь), а остальное количество - из монопрепарата синтетической фолиевой кислоты [13].

После 12 нед. беременности рекомендуется возврат к физиологическим дозировкам (менее 1 мг/сут), учитывая имеющиеся данные о неблагоприятном влиянии на здоровье матери и ее ребенка длительного приема высоких доз фолиевой кислоты. С избытком синтетических фолатов ассоциирован риск злокачественных, сердечно-сосудистых заболеваний и когнитивных расстройств $[35,36]$. Высокое потребление синтетических фолатов матерями во время беременности также является фактором риска инфекционно-воспалительных и аллергических заболеваний у детей, инсулинорезистентности, злокачественных заболеваний, нарушения когнитивных способностей и зрения [37-39].

Мужчинам в период планирования зачатия ребенка, так же как и будущей матери, рекомендовано принимать профилактическую дозу фолиевой кислоты. У мужчин фолиевая кислота влияет на количество и генетическое качество сперматозоидов. Регулярное употребление фолиевой кислоты препятствует анеуплоидии, которая может быть причиной бесплодия, выкидышей и рождения детей с синдромом Дауна, Шерешевского - Тернера, Кляйнфельтера и др. [40].

\section{Слишком короткий промежуток между} последовательными родами, как и чрезмерное удлинение интергенетического интервала, значительно повышает риск ВПР, перинатальной смертности, преждевременных родов, рождения маловесных детей, материнской и неонатальной заболеваемости. Единственный путь гарантированного соблюдения оптимального интервала между родами - рациональная контрацепция

В рекомендациях FIGO 2015 г. [17] делается особый акцент на важности просветительской деятельности врачей. Необходимо сообщать женщинам репродуктивного возраста о пользе регулярного приема фолатов, особенно при планировании беременности и при отсутствии надежной контрацепции, используя для этого любое обращение женщины к медицинским работникам (профилактические осмотры, консультации по планированию беременности, контрацепции, профилактике онкологических заболеваний и т. д.).

Дополнительный прием йода. Всем женщинам, планирующим беременность, необходимо потребление йода не менее 150 мкг/сут. При проживании в эндемичном по йододефициту районе рекомендуется его дополнительная дотация [2, 41]. В популяциях с йододефицитом отмечается высокий уровень эндемического кретинизма, врожденных заболеваний щитовидной железы. Дополнительный прием препаратов йода в периконцепционный период снижает показатели неонатальной и младенческой смертности и улучшает психосоматическое развитие детей после рождения.

\section{В челом прегравидарное консультирование следует рассматривать как реализацию конституционного права каждого гражданина РФ на охрану здоровья и медицинскую помощь, а также меру государственной поддержки семьи, материнства и детства}

Профилактика железодефицитной анемии. Женщины репродуктивного возраста наиболее часто страдают от дефицита железа и анемии. К группе риска развития анемии ВОЗ относит абсолютно всех менструирующих женщин независимо от характера кровотечений [42]. По обобщенным данным, латентный дефицит железа имеют от 60 до 85\% женщин нашей страны [43]. Анемия во время беременности повышает риск преждевременных родов, рождения маловесных детей, материнской и неонатальной смертности, гнойно-септических осложнений и кровотечений после родов [44]. Недостаток железа также неблагоприятно влияет на нейрогенез плода. Вследствие гипоксии и снижения интенсивности энергетического обмена происходит недостаточное ветвление дендритов, уменьшается количество и сложность нейронных сетей коры головного мозга, нарушается обмен дофамина и норадреналина (отвечающих за циклы сна и бодрствования, обучения, памяти), нарушаются процессы миелинизации нейронов, что в конечном итоге неблагоприятно отражается на умственном и психическом развитии ребенка $[45,46]$.

Следуя обновленным рекомендациям ВО3 [42], всем менструирующим женщинам и девочкам-подросткам в регионах с частотой анемии более $40 \%$ необходимо принимать профилактическую дозу железа (30-60 мг элементарного железа) ежедневно в течение трех последовательных месяцев в году. Кроме того, по мнению экспертов ВОЗ, ежедневный прием железа и фолиевой кислоты должен быть рутинной практикой антенатального наблюдения беременных и продолжаться в течение 3 мес. после родов. Дотацию железа рекомендуется проводить с помощью препаратов железа, фортифицированных железом продуктов питания или назначением ВМК, содержащих железо. 
Дотация полиненасыщенных жирных кислот (ПНЖК). Для всех женщин в периконцепционный период желательно дополнительное поступление ПНЖК в дозировке 200-300 мг/сут с последующим продолжением во время гестации для профилактики осложнений беременности и обеспечения физиологического формирования структур головного мозга, органа зрения и иммунной системы плода. Оптимальный способ увеличения поступления ПНЖК - расширение диеты, при невозможности - медикаментозно [47, 48].

При дотации витаминов и минеральных веществ необходимо учитывать возможные негативные последствия их избыточного потребления (тератогенность, риск многоплодия, аллергические реакции, снижение функции щитовидной железы, токсическое воздействие на печень и желудочно-кишечный тракт).

\section{III. ДОПОЛНИТЕЛЬНЫЕ МЕРОПРИЯТИЯ У ЖЕНЩИН С ОТЯГОЩЕННЫМ АКУШЕРСКО- ГИНЕКОЛОГИЧЕСКИМ АНАМНЕЗОМ И ЭКСТРАГЕНИТАЛЬНЫМИ ЗАБОЛЕВАНИЯМИ}

У женщин, имеющих гинекологические и соматические заболевания, значительно не только ухудшен прогноз наступления и вынашивания беременности, но и повышен риск для здоровья, связанный с беременностью [41].

\section{Анемия во время беременности повышает риск преждевременных родов, рождения маловесных детей, материнской и неонатальной смертности, гнойно-септических осложнений и кровотечений после родов}

Диагностические и лечебно-профилактические мероприятия у женщин с экстрагенитальными заболеваниями проводятся совместно с врачами смежных специальностей. Тактика регламентирована Приказом №572н от 1 ноября 2012 г. с изменениями и дополнениями, внесенными в пункт 15 Приказом №5н от 12 января 2016 г. Планирование беременности возможно только после коллегиального решения специалистов с учетом пожеланий пациентки.

Коррекция выявленных соматических и гинекологических заболеваний в период планирования беременности обеспечивает профилактику осложнений беременности и улучшает прогноз рождения здорового ребенка. Психологическое сопровождение пациенток, переживших критические акушерские состояния (near miss), редуцирует негативные последствия психотравмирующего опыта предыдущей беременности и формирует благоприятную установку на реализацию репродуктивной функции [49].

\section{По мнению экспертов В03, ежедневный прием железа и фолиевой кислоты должен быть рутинной практикой антенатального наблюдения беременных и продолжаться в течение 3 мес. после родов}

В клиническом протоколе подробно изложены основные лечебно-профилактические мероприятия и рекомендации по планированию беременности, необходимые женщинам с эктрагенитальными заболеваниями (эндокринными, мочевой системы, сердечнососудистыми, желудочно-кишечными, анемией) и отягощенным гинекологическим анамнезом (СПКЯ, ВЗОМТ, эндометриозом, рубцом на матке после кесарева сечения, цервиковагинальными заболеваниями, с нарушениями менструального цикла, невынашиванием беременности, бесплодием) [3].

Мероприятия, противопоказанные в период прегравидарной подготовки

1. Избыточные назначения. Излишне глубокое обследование супружеских пар молодого возраста при отсутствии факторов риска, равно как и неоправданно широкий спектр медикаментозной профилактики и лечения, приравнивают к дефектам оказания медицинской помощи. Необходимость диагностических, профилактических и лечебных мероприятий, проводимых в рамках прегравидарной подготовки, должна быть обоснована с позиций доказательной медицины.

2. Полипрагмазия.

3. Профилактическая санация влагалища

Таким образом, прегравидарное консультирование необходимо проводить всем женщинам репродуктивного возраста на любом плановом и внеплановом визите к акушеру-гинекологу. Прегравидарная подготовка должна стать необходимой составной частью медицинского наблюдения всех женщин репродуктивного возраста, такой же, как обследование их во время беременности.

\section{ЛИТЕРАТУРА}

1. Dean SV, Lassi ZS, Imam AM et al. Preconception care: closing the gap in the continuum of care to accelerate im- provements in maternal, newborn and child health. Reprod. Health., 2014, 11(Suppl. 3): S1.
2. Berghella V. Preconceptional care: in book Obstetric Evidence Based Guidelines. 2nd Edition. Ed. by Berghella V. 2012: P1-11.

3. Прегравидарная подготовка: клинический протокол. [авт. разраб. В.Е. Радзинский и др.]. М.: Редакция журнала StatusPraesens, 2016. 80 с.
4. Радзинский В.Е., Пустотина О.А. Планирование семьи в XXI веке. М.: ГЭОТАР- Медиа, 2015. С. 17.

5. De Franco EA, Seske LM, Greenberg JM et al. Influ- ence of interpregnancy interval on neonatal morbidity. Am. J. Obstet. Gynecol., 2015, 212(3): 386.e1-386.e9. [PMID: 25460837] 
6. Chen I, Jhangri GS, Chandra S. Relationship between in-terpregnancy interval and congenital anomalies. Am. J. Obstet. Gynecol., 2014 210(6): 564.e1-564.e8. [PMID: 24508646]

7. RCOG: Best practice in postpartum family planning. Best Practice Paper No1. 2015.13 P. URL: https://www.rcog.org. uk/en/guidelines-research-services/guidelines/bpp1/.

8. Love ER, Bhattacharya S, Smith NC et al. Effect of in- terpregnancyinterva lon outcomes of pregnancy after miscar-riage: Retrospective analysis of hospital episode statistics in Scotland. BMJ, 2010, 341: 3967.

9. CHOICE - итоги первой общеевропейской программы по контрацепции. URL: http:// www.epochtimes.ru/content/ view/38373/7.

10. Радзинский В.Е. Акушерская агрессия. М.: Изд-во журнала StatusPraesens, 2011. 688 с.

11. Ciancimino L, Laganà AS, Chiofalo $B$ et al. Would it be too late? A retrospective casecontrol analysis to evaluate maternal-fetal outcomes in advanced maternal age. Arch. Gynecol. Obstet., 2014, 290(6): 1109-1114.

12. Гипертензивные расстройства во время беременности, в родах и послеродовом периоде. Преэклампсия. Эклампсия. Клинические рекомендации МЗ РФ (протокол лечения). М., 2016.72 с

13. Hanson MA, Bardsley A, De-Regil LM, et al. Think Nutrition First. Intern J Gynecol Obstetr, 2015, 131(4): 213-253.

14. Devakumar D, Fall CHD, Sachdev HS et al. Maternal antenatal multiple micronutrient supplementation for long- term health benefits in children: a systematic review and meta-analysis. BMC Medicine, 2016, 14: 90.

15. Gernand AD, Schulze KJ, Stewart CP et al. Micronutrient deficiencies in pregnancy worldwide: health effects and prevention. Nat. Rev. Endocrinol., 2016, 12(5): 274-289. [PMID: 27032981]

16. McLean HO, Fiebelkorn AP, Temte JL et al. Centers for disease control and prevention: Prevention of measles, rubella, congenital rubella syndrome, and mumps, 2013: summary recommendations of the Advisory Committee on Immunization Practices (ACIP). MMWR Recomm Rep, 2013, 62(RR-04): 1-4.

17. Best practice in maternal-fetal medicine. FIGO Working Group on Best Practice in Maternal-Fetal Medicine. Int. J. Gynecol. Obstet., 2015, 128: 80-82.

18. Демикова Н.С., Лапина А.С., Подольная М.А. и др. Динамика частоты врожденных пороков развития в РФ (по данным федеральной базы мониторинга ВПР за 2006-2012 гг.). Российский вестник перинатологии и педиатрии, 2015, 2: 72-77.

19. Greenberg JA, Bell SJ, Guan Y et al. Folic Acid Supplementaion and Pregnancy: More Than Just Neural Tube Defect Prevention. Rev Obstet Gynecol, 2011, 4(2): 52-59.

20. Федеральная служба государственной статистики (Росстат): Российский статистический ежегодник - 2015: Статистический сборник.

21. De-Regil LM, Pena-Rosas JP, FernandezGaxiola AC, Rayco-Solon P. Effects and safety of periconceptional oral folate supplementation for preventing birth defects. Cochr Database Syst Rev, 2015, CD007950.

22. Van Beynum IM et al. Protective effect of periconceptional folic acid supplements on the risk of congenital heart defects: a registrybased case-control study in the northern Netherlands. Eur Heart J, 2010, 31(4): 464-471.

23. Czeizel AE, Puho EH, Langmar $\mathrm{Z}$ et al. Possible association of folic acid supplemen tation during pregnancy with reduction of preterm birth: a population-based study. Eur J Obstet Gynecol Reprod Biol, 2010, 148: 135140

24. Bukowski R, Malone FD, Porter FT et al. Preconceptional folate supplementation and the risk of spontaneous preterm birth: a cohort study. PLoS Med, 2009, 6: e1000061.

25. Timmermans $S$, Jaddoe VW, Hofman A et al. Periconception folic acid supplementation, fetal growth and the risks of low birth weight and preterm birth: the Generation $\mathrm{R}$ Study. Br J Nutr, 2009, 102: 777-785.

26. Hodgetts VA, Morris RK, Francis A, et al. Effectiveness of folic acid supplementation in pregnancy on reducing the risk of smallfor-gestational age neonates: a population study, systematic review and meta-analysis. BJOG, 2014, DOI: 10.1111/1471-0528.13202.

27. Smith $A D$, Smith $S M$, de Jager $C A$, et al. Homocysteine-lowering by $B$ vitamins slows the rate of accelerated brain atrophy in mild cognitive impairment. A randomized controlled trial. PLOS ONE, 2010, 5: e12244.

28. Julvez J, Fortuny J, Mendez M, Torrent M, Ribas-Fito N, Sunyer J. Maternal use of folic acid supplements during pregnancy and four-year-old neurodevelopment in a population-based birth cohort. Paediatr Perinat Epidemiol, 2009, 23: 199-206.

29. Roth C, Magnus P, Schjolberg S, Stoltenberg C, Suren P, McKeague IW et al. Folic acid supplements in pregnancy and severe language delay in children. JAMA, 2011, 306: 1566-1573.

30. Pietrzik K, Lamers Y, Brämswig S, PrinzLangenohl R. Calculation of red blood cell folate steady state conditions and elimination kinetics after daily supplementation with various folate forms and doses in women in childbearing age. Am J Clin Nutr, 2007, 86: 1414-1419.

31. Brämswig $S$, Prinz-Langenohl R, Lamers $Y$ et al. Supplementation with a multivitamin containing $800 \mu \mathrm{g}$ of folic acid shortens the time to reach the preventive red blood cell folate concentration in healthy women. Int J Vitam Nutr Res, 2009, 79: 61-70.

32. Czeizel AE, Dudás I. Prevention of the first occurrence of neural-tube defects by periconceptional vitamin supplementation. N Engl J Med, 1992, 327: 1832-5.

33. Goh YI, Bollano E, Einarson TR, Koren G. Prenatal multivitamin supplementation and rates of congenital anomalies: a meta-analysis. J Obstet Gynaecol Can, 2006, 28(8): 680.

34. Wilson RD et al. Pre-conception Folic Acid and Multivitamin Supplementation for the
Primary and Secondary Prevention of Neural Tube Defects and Other Folic Acid-Sensitive Congenital Anomalies. J. Obstet. Gynaecol. Can., 2015, 37(6): 534-352.

35. Kim YI. Folate and colorectal cancer: an evidence-based critical review. Mol Nutr Food Re, 2007, 51(3): 267-292.

36. Morris MS, Jacques PF, Rosenberg IH, Selhub J. Circulating unmetabolized folic acid and 5-methyltetrahydrofolate in relation to anemia, macrocytosis, and cognitive test performance in American seniors. Am J Clin Nutr 2010, 91: 1733-1744.

37. Linabery AM, Johnson KJ, Ross JA. Childhood cancer incidence trends in association with US folic acid fortification (19862008). Pediatrics, 2012, 129: 1125-1133.

38. Bekkers MB, Elstgeest LE, Scholtens S et al. Maternal use of folic acid supplements during pregnancy and childhood respiratory health and atopy: the PIAMA birth cohort study. Eur Respir J, 2011.

39. Dunstan JA, West C, McCarthy S et al. The relationship between maternal folate status in pregnancy, cord blood folate levels, and allergic outcomes in early childhood. Allergy, 2011, 67(1): 50-57.

40. van der Zee B, de Wert G, Steegers EA et al. Ethical aspects of paternal preconception lifestyle modification. Am J Obstet Gynacol, 2013, 209(1): 11

41. Maternal-Fetal Evidence Based Guidelines. 2nd Edition. Ed. by Berghella V. 2012. 462 P

42. Guideline: Daily iron supplementation in adult women and adolescent girls. Geneva: World Health Organization; 2016.

43. Стуклов Н.И., Козинец Г.И., Леваков С.А. Огурцов П.П. Анемии при гинекологических и онкогинекологических заболеваниях. М. Медицинское информационное агентство, 2013. 240 c.

44. International Anemia Consultative Group. Report of the 2001 International Anemia Consultative Group Symposium. Why is iron important and what to do about it: a new perspective. Washington, DC, INACG Secretariat, 2002: 1-50.

45. Beard JL. Why iron deficiency is important in infant development. J Nutr, 2008, 138(12): 2534- 6

46. Bresgen N, Eckl PM. Oxidative Stress and the Homeodynamics of Iron Metabolism. Biomolecules, 2015, 5(2): 808-847.

47. Innis SM. Metabolic programming of longterm outcomes due to fatty acid nutrition in early life. Matern. Child. Nutr., 2011, 7(Suppl. 2): $112-123$

48. Hansen S, Strøm M, Maslova E et al. Fish oil supplementation during pregnancy and allergic respiratory disease in the adult offspring. J. Allergy Clin. Immunol., 2016.

49. Лебеденко E.Ю. Near miss: на грани материнских потерь. Под ред. В.Е. Радзинского. М.: Изд-во журнала StatusPraesens, 2015. 184 с. 